\title{
GRADIENT ESTIMATES FOR A NONLINEAR ELLIPTIC EQUATION ON COMPLETE RIEMANNIAN MANIFOLDS
}

\author{
BINGQING MA, GUANGYUE HUANG, AND YONG LUO
}

\begin{abstract}
In this short note, we consider gradient estimates for positive solutions to the following nonlinear elliptic equation on a complete Riemannian manifold:$$
\Delta u+c u^{\alpha}=0,
$$

where $c, \alpha$ are two real constants and $c \neq 0$.
\end{abstract}

\section{Introduction}

It is well-known that for complete noncompact Riemannian manifolds with nonnegative Ricci curvature, Yau [1] has proved that any positive or bounded solution to the equation

$$
\Delta u=0
$$

must be constant. In [1, Brighton studied $f$-harmonic function on a mooth metric measure space. That is, he consider positive solutions to the equation

$$
\Delta_{f} u=0
$$

and obtain some similar results to Yau's under the Bakry-Émery Ricci curvature condition.

It is easy to see that the equation (1.1) can be seen as a special case of

$$
\Delta u+c u^{\alpha}=0
$$

with $c, \alpha$ two real constants. In particular, if $c=0$ in (1.3), then the equation (1.3) becomes (1.1). If $c<0$ and $\alpha<0$, the equation (1.3) on a bounded smooth domain in $\mathbb{R}^{n}$ is known as the thin film equation, which describes a steady state of the thin film (see [3]). For $c$ a function, the equation (1.3) is studied by Gidas and Spruck in [2] with $1 \leq \alpha \leq \frac{n+2}{n-2}$ when $n>2$ and lather it is studied by $\mathrm{Li}$ in [7] to achieve gradient estimates and Liouville type results with $1<\alpha<\frac{n}{n-2}$ when $n>3$. In particular, Li achieved a gradient estimate for positive solution of (1.3) when $c$ is a positive constant and $1<\alpha<\frac{n}{n-2}$.

2010 Mathematics Subject Classification. Primary 58J35, Secondary 35B45.

Key words and phrases. Gradient estimate, nonlinear elliptic equation, Liouville-type theorem.

The research of the authors is supported by NSFC Nos. 
Therefore, it is natural to try to achieve gradient estimates for positive solutions to the nonlinear elliptic equation (1.3) with other $c \neq 0$ and $\alpha$. In this direction Yang in [10] proved the following result:

Theorem 1.1 (Yang). Let $M$ be a complete noncompact Riemannian manifold of dimension $n$ without boundary. Let $B_{p}(2 R)$ be a geodesic ball of radius $2 R$ around $p \in M$. We denote $-K(2 R)$ with $K(2 R) \geq 0$ such that $\operatorname{Ric}_{i j}\left(B_{p}(2 R)\right) \geq-K g_{i j}$. Suppose that $u(x)$ is a positive smooth solution of the equation (1.3) with $\alpha<0$. Then we have

(i) If $c>0$, then $u(x)$ satisfies the estimate

$$
\frac{|\nabla u|^{2}}{u^{2}}+c u^{\alpha-1} \leq C(n, \alpha)\left(K+\frac{1}{R^{2}}(1+\sqrt{K} R \operatorname{coth}(\sqrt{K} R))\right)
$$

on $B_{p}(R)$ and $C(n, \alpha)$ is a positive constant which depends on $n, \alpha$.

(ii) If $c<0$, then $u(x)$ satisfies the estimate

$\frac{|\nabla u|^{2}}{u^{2}}+c u^{\alpha-1} \leq C(n, \alpha)\left(|c|\left(\inf _{B_{p}(2 R)} u\right)^{\alpha-1}+K+\frac{1}{R^{2}}(1+\sqrt{K} R \operatorname{coth}(\sqrt{K} R))\right)$ on $B_{p}(R)$ and $C(n, \alpha)$ is a positive constant which depends on $n, \alpha$.

After studying Yang's argument carefully, we find in the case of $c>0$, the gradient estimate in (i) actually holds when $\alpha \leq 1$, that is we have

Theorem 1.2. Let $M$ be a complete noncompact Riemannian manifold of dimension $n$ without boundary. Let $B_{p}(2 R)$ be a geodesic ball of radius $2 R$ around $p \in M$. We denote $-K(2 R)$ with $K(2 R) \geq 0$ such that $\operatorname{Ric}_{i j}\left(B_{p}(2 R)\right) \geq-K g_{i j}$. Suppose that $u(x)$ is a positive smooth solution of the equation (1.3) with $\alpha \leq 1$ and $c>0$. Then we have

$$
\frac{|\nabla u|^{2}}{u^{2}}+c u^{\alpha-1} \leq C(n, \alpha)\left(K+\frac{1}{R^{2}}(1+\sqrt{K} R \operatorname{coth}(\sqrt{K} R))\right)
$$

on $B_{p}(R)$ and $C(n, \alpha)$ is a positive constant which depends on $n, \alpha$.

The proof of the above theorem is the same as Yang's proof of theorem 1.1. and we will only give a sketch of it in the appendix. As a corollary of the above theorem we have the following Liouville type result:

Corollary 1.3. Let $M$ be a complete noncompact Riemannian manifold of dimension $n$ without boundary. Suppose that the Ricci curvature of $M$ is nonnegative. Then there does not exist a positive solution to equation (1.3) with $\alpha \leq 1$ and $c>0$.

Suppose that $u(x)$ is a positive solution to equation (1.3). Following Brighton's argument in [1] by choosing a test function $u^{\epsilon}(\epsilon \neq 0)$, we can also get the following gradient estimate to $u(x)$.

Theorem 1.4. Let $(M, g)$ be an $n$-dimensional complete Riemannian manifold with $R_{i j}\left(B_{p}(2 R)\right) \geq-K g_{i j}$, where $K \geq 0$ is a constant. If $u$ is a positive solution to (1.3) on $B_{p}(2 R)$ with $c$ and $\alpha$ satisfying one of the following two cases: 
(1) $c<0$ and $\alpha>0$;

(2) $c>0$ and $\frac{n+2}{2(n-1)}<\alpha<\frac{2 n^{2}+9 n+6}{2 n(n+2)}$ with $n \geq 3$,

then we have for any $x \in B_{p}(R)$

$$
|\nabla u(x)| \leq C(n, \alpha) M \sqrt{K+\frac{1}{R^{2}}(1+\sqrt{K} R \operatorname{coth}(\sqrt{K} R))},
$$

where $M=\sup _{x \in B_{p}(2 R)} u(x)$ and the positive constant $C(n, \alpha)$ depends only on $n, \alpha$.

Remark 1.1. In case (2), compared with Li's gradient estimate in [7] our right range for $\alpha$ is bigger than $\frac{n}{n-2}$ when $n \geq 13$.

Letting $R \rightarrow \infty$ in (1.4), we obtain the following gradient estimates on complete noncompact Riemannian manifolds:

Corollary 1.5. Let $\left(M^{n}, g\right)$ be an n-dimensional complete noncompact Riemannian manifold with $R_{i j} \geq-K g_{i j}$, where $K \geq 0$ is a constant. Suppose that $u$ is a positive solution to (1.3) such that $c, \alpha$ satisfies one of the two cases given in Theorem 1.4. Then we have

$$
|\nabla u| \leq C(n, \alpha) M \sqrt{K}
$$

where $M=\sup _{x \in M} u(x)$.

Remark 1.2. Recently, using the ideas of Brighton in [1], some Liouville type results are achieved to positive solutions of the nonlinear elliptic equation

$$
\Delta u+a u \log u=0
$$

in [4](for more developments, see [6, 8]), and for porous medium and fast diffusion equations in [5].

Acknowledgment. Yong Luo would like to thank Dr. Linlin Sun for his stimulating discussions on this problem.

\section{Proof of theorem 1.4}

Let $h=u^{\epsilon}$, where $\epsilon \neq 0$ is a constant to be determined. Then we have

$$
\begin{aligned}
\Delta h & =\epsilon(\epsilon-1) u^{\epsilon-2}|\nabla u|^{2}+\epsilon u^{\epsilon-1} \Delta u \\
& =\epsilon(\epsilon-1) u^{\epsilon-2}|\nabla u|^{2}-c \epsilon u^{\alpha+\epsilon-1} \\
& =\frac{\epsilon-1}{\epsilon} \frac{|\nabla h|^{2}}{h}-c \epsilon h^{\frac{\alpha+\epsilon-1}{\epsilon}},
\end{aligned}
$$


where in the second equality of (2.1), we used (1.3). Hence, we have

$$
\begin{aligned}
\nabla h \nabla \Delta h & =\nabla h \nabla\left(\frac{\epsilon-1}{\epsilon} \frac{|\nabla h|^{2}}{h}-c \epsilon h^{\frac{\alpha+\epsilon-1}{\epsilon}}\right) \\
& =\frac{\epsilon-1}{\epsilon} \nabla h \nabla \frac{|\nabla h|^{2}}{h}-c(\alpha+\epsilon-1) h^{\frac{\alpha+\epsilon-1}{\epsilon}} \frac{|\nabla h|^{2}}{h} \\
& =\frac{\epsilon-1}{\epsilon h} \nabla h \nabla\left(|\nabla h|^{2}\right)-\frac{\epsilon-1}{\epsilon} \frac{|\nabla h|^{4}}{h^{2}}-c(\alpha+\epsilon-1) h^{\frac{\alpha+\epsilon-1}{\epsilon}} \frac{|\nabla h|^{2}}{h} .
\end{aligned}
$$

Applying (2.1) and (2.2) into the well-known Bochner formula to $h$, we have

$$
\begin{aligned}
\frac{1}{2} \Delta|\nabla h|^{2}= & \left|\nabla^{2} h\right|^{2}+\nabla h \nabla \Delta h+\operatorname{Ric}(\nabla h, \nabla h) \\
\geq & \frac{1}{n}(\Delta h)^{2}+\nabla h \nabla \Delta h-K|\nabla h|^{2} \\
= & \frac{1}{n}\left(\frac{\epsilon-1}{\epsilon} \frac{|\nabla h|^{2}}{h}-c \epsilon h^{\frac{\alpha+\epsilon-1}{\epsilon}}\right)^{2}+\frac{\epsilon-1}{\epsilon} \frac{\nabla h}{h} \nabla\left(|\nabla h|^{2}\right) \\
& -\frac{\epsilon-1}{\epsilon} \frac{|\nabla h|^{4}}{h^{2}}-c(\alpha+\epsilon-1) h^{\frac{\alpha+\epsilon-1}{\epsilon}} \frac{|\nabla h|^{2}}{h}-K|\nabla h|^{2} \\
= & \left(\frac{(\epsilon-1)^{2}}{n \epsilon^{2}}-\frac{\epsilon-1}{\epsilon}\right) \frac{|\nabla h|^{4}}{h^{2}}-c\left[\frac{n+2}{n}(\epsilon-1)+\alpha\right] h^{\frac{\alpha+\epsilon-1}{\epsilon}} \frac{|\nabla h|^{2}}{h} \\
& +\frac{c^{2} \epsilon^{2}}{n} h^{\frac{2(\alpha+\epsilon-1)}{\epsilon}}+\frac{\epsilon-1}{\epsilon} \frac{\nabla h}{h} \nabla\left(|\nabla h|^{2}\right)-K|\nabla h|^{2} .
\end{aligned}
$$

By analyzing (2.3) we have the following lemmas.

Lemma 2.1. Let $u$ be a positive solution to (1.3) and $R_{i j} \geq-K g_{i j}$ for some nonnegative constant $K$. Denote $h=u^{\epsilon}$ with $\epsilon \neq 0$. If $c<0$ and $\alpha>0$, then there exists $\epsilon \in(0,1)$ such that

$$
\begin{aligned}
\frac{1}{2} \Delta|\nabla h|^{2} \geq & \left(\frac{(\epsilon-1)^{2}}{n \epsilon^{2}}-\frac{\epsilon-1}{\epsilon}\right) \frac{|\nabla h|^{4}}{h^{2}} \\
& +\frac{\epsilon-1}{\epsilon} \frac{\nabla h}{h} \nabla\left(|\nabla h|^{2}\right)-K|\nabla h|^{2} .
\end{aligned}
$$

Proof. In (2.3), if $c<0$ and $\alpha>0$, we can choose $\epsilon \in(0,1)$ close enough to 1 such that

$$
-c\left[\frac{n+2}{n}(\epsilon-1)+\alpha\right] \geq 0,
$$

and then (2.4) follows directly.

Lemma 2.2. Let $u$ be a positive solution to (1.3) and $R_{i j} \geq-K g_{i j}$ for some nonnegative constant $K$. Denote $h=u^{\epsilon}$ with $\epsilon \neq 0$. If $c>0$ and for a fixed $\alpha$, there exist two positive constants $\epsilon, \delta$ such that

$$
c\left[\frac{n+2}{n}(\epsilon-1)+\alpha\right]>0
$$


and

$$
\frac{c^{2} \epsilon^{2}}{n}-\frac{c}{\delta}\left(\frac{n+2}{n}(\epsilon-1)+\alpha\right)>0
$$

then we have

$$
\begin{aligned}
\frac{1}{2} \Delta|\nabla h|^{2} \geq & {\left[\frac{(\epsilon-1)^{2}}{n \epsilon^{2}}-\frac{\epsilon-1}{\epsilon}-\delta c\left(\frac{n+2}{n}(\epsilon-1)+\alpha\right)\right] \frac{|\nabla h|^{4}}{h^{2}} } \\
& +\frac{\epsilon-1}{\epsilon} \frac{\nabla h}{h} \nabla\left(|\nabla h|^{2}\right)-K|\nabla h|^{2} .
\end{aligned}
$$

Proof. For a fixed point $p$, if there exists a positive constant $\delta$ such that $h^{\frac{\alpha+\epsilon-1}{\epsilon}} \leq \delta \frac{|\nabla h|^{2}}{h}$, according to (2.5), then (2.3) becomes

$$
\begin{aligned}
\frac{1}{2} \Delta|\nabla h|^{2} \geq & {\left[\frac{(\epsilon-1)^{2}}{n \epsilon^{2}}-\frac{\epsilon-1}{\epsilon}-\delta c\left(\frac{n+2}{n}(\epsilon-1)+\alpha\right)\right] \frac{|\nabla h|^{4}}{h^{2}} } \\
& +\frac{c^{2} \epsilon^{2}}{n} h^{\frac{2(\alpha+\epsilon-1)}{\epsilon}}+\frac{\epsilon-1}{\epsilon} \frac{\nabla h}{h} \nabla\left(|\nabla h|^{2}\right)-K|\nabla h|^{2} \\
\geq & {\left[\frac{(\epsilon-1)^{2}}{n \epsilon^{2}}-\frac{\epsilon-1}{\epsilon}-\delta c\left(\frac{n+2}{n}(\epsilon-1)+\alpha\right)\right] \frac{|\nabla h|^{4}}{h^{2}} } \\
& +\frac{\epsilon-1}{\epsilon} \frac{\nabla h}{h} \nabla\left(|\nabla h|^{2}\right)-K|\nabla h|^{2} .
\end{aligned}
$$

On the contrary, at the point $p$, if $h^{\frac{\alpha+\epsilon-1}{\epsilon}} \geq \delta \frac{|\nabla h|^{2}}{h}$, then (2.3) becomes

$$
\begin{aligned}
\frac{1}{2} \Delta|\nabla h|^{2} \geq & \left(\frac{(\epsilon-1)^{2}}{n \epsilon^{2}}-\frac{\epsilon-1}{\epsilon}\right) \frac{|\nabla h|^{4}}{h^{2}}+\left[\frac{c^{2} \epsilon^{2}}{n}-\frac{c}{\delta}\left(\frac{n+2}{n}(\epsilon-1)+\alpha\right)\right] h^{\frac{2(\alpha+\epsilon-1)}{\epsilon}} \\
& +\frac{\epsilon-1}{\epsilon} \frac{\nabla h}{h} \nabla\left(|\nabla h|^{2}\right)-K|\nabla h|^{2} \\
\geq & \left.\left(\frac{(\epsilon-1)^{2}}{n \epsilon^{2}}-\frac{\epsilon-1}{\epsilon}\right)+\delta^{2}\left[\frac{c^{2} \epsilon^{2}}{n}-\frac{c}{\delta}\left(\frac{n+2}{n}(\epsilon-1)+\alpha\right)\right]\right\} \frac{|\nabla h|^{4}}{h^{2}} \\
& +\frac{\epsilon-1}{\epsilon} \frac{\nabla h}{h} \nabla\left(|\nabla h|^{2}\right)-K|\nabla h|^{2} \\
\geq & \left.\frac{(\epsilon-1)^{2}}{n \epsilon^{2}}-\frac{\epsilon-1}{\epsilon}-\delta c\left(\frac{n+2}{n}(\epsilon-1)+\alpha\right)\right] \frac{|\nabla h|^{4}}{h^{2}} \\
& +\frac{\epsilon-1}{\epsilon} \frac{\nabla h}{h} \nabla\left(|\nabla h|^{2}\right)-K|\nabla h|^{2}
\end{aligned}
$$

as long as

$$
\frac{c^{2} \epsilon^{2}}{n}-\frac{c}{\delta}\left(\frac{n+2}{n}(\epsilon-1)+\alpha\right)>0
$$

In both cases, (2.7) holds always. We complete the proof of Lemma 2.2 ,

In order to obtain the upper bound of $|\nabla h|$ by using the maximum principle, it is sufficient to choose the coefficient of $\frac{|\nabla h|^{4}}{h^{2}}$ in (2.4) and (2.7) such 
that it is positive. In case of Lemma 2.2, we need to choose appropriate $\epsilon, \delta$ such that

$$
\frac{(\epsilon-1)^{2}}{n \epsilon^{2}}-\frac{\epsilon-1}{\epsilon}-\delta c\left(\frac{n+2}{n}(\epsilon-1)+\alpha\right)>0 .
$$

Under the assumption of (2.5), the inequality (2.6) becomes

$$
\delta>\frac{n c}{c^{2} \epsilon^{2}}\left(\frac{n+2}{n}(\epsilon-1)+\alpha\right)
$$

and (2.11) becomes

$$
\delta<\frac{\frac{(\epsilon-1)^{2}}{n \epsilon^{2}}-\frac{\epsilon-1}{\epsilon}}{c\left(\frac{n+2}{n}(\epsilon-1)+\alpha\right)} .
$$

In order to ensure we can choose a positive $\delta$, from (2.12) and (2.13), we need choose an $\epsilon$ satisfying

$$
\frac{n c}{c^{2} \epsilon^{2}}\left(\frac{n+2}{n}(\epsilon-1)+\alpha\right)<\frac{\frac{(\epsilon-1)^{2}}{n \epsilon^{2}}-\frac{\epsilon-1}{\epsilon}}{c\left(\frac{n+2}{n}(\epsilon-1)+\alpha\right)} .
$$

In particular, (2.14) can be written as

$$
\begin{aligned}
n^{2}\left(\frac{n+2}{n}(\epsilon-1)+\alpha\right)^{2} & <n \epsilon^{2}\left(\frac{(\epsilon-1)^{2}}{n \epsilon^{2}}-\frac{\epsilon-1}{\epsilon}\right) \\
& =(\epsilon-1)^{2}-n \epsilon(\epsilon-1),
\end{aligned}
$$

which is equivalent to

$$
\begin{aligned}
{\left[n^{2}+5 n+3\right] \epsilon^{2} } & +\left[2(\alpha-1)\left(n^{2}+2 n\right)-(5 n+6)\right] \epsilon \\
+ & (\alpha-1)^{2} n^{2}-4(\alpha-1) n+3<0 .
\end{aligned}
$$

By a direct calculation, under the condition

$$
\frac{-(n-4)-\sqrt{n^{2}+5 n+3}}{2(n-1)}<\alpha-1<\frac{-(n-4)+\sqrt{n^{2}+5 n+3}}{2(n-1)},
$$

we have

$$
\begin{aligned}
{[} & \left.2(\alpha-1)\left(n^{2}+2 n\right)-(5 n+6)\right]^{2}-4\left[n^{2}+5 n+3\right]\left[(\alpha-1)^{2} n^{2}-4(\alpha-1) n+3\right] \\
= & 4(\alpha-1)^{2}\left[\left(n^{2}+2 n\right)^{2}-n^{2}\left(n^{2}+5 n+3\right)\right]+4(\alpha-1)\left[4 n\left(n^{2}+5 n+3\right)\right. \\
& \left.-\left(n^{2}+2 n\right)(5 n+6)\right]+(5 n+6)^{2}-12\left(n^{2}+5 n+3\right) \\
= & 4(\alpha-1)^{2}\left[-n^{3}+n^{2}\right]+4(\alpha-1)\left[-n^{3}+4 n^{2}\right]+13 n^{2} \\
= & n^{2}\left\{-4(n-1)(\alpha-1)^{2}-4(n-4)(\alpha-1)+13\right\} \\
> & 0,
\end{aligned}
$$

which shows the quadratic inequality (2.16) with respect to $\epsilon$ has two real roots.

Now we are ready to prove the following proposition. 
Proposition 2.3. Let $u$ be a positive solution to (1.3) and $R_{i j} \geq-K g_{i j}$ for some nonnegative constant $K$. If we choose $c$ and $\alpha$ satisfies one of the following two cases:

(1) $c<0$ and $\alpha>0$;

(2) $c>0$ and $\frac{n+2}{2(n-1)}<\alpha<\frac{2 n^{2}+9 n+6}{2 n(n+2)}$ with $n \geq 3$, Then we have

$$
\frac{1}{2} \Delta|\nabla h|^{2} \geq C_{1}(n, \alpha) \frac{|\nabla h|^{4}}{h^{2}}-C_{2}(n, \alpha) \frac{\nabla h}{h} \nabla\left(|\nabla h|^{2}\right)-K|\nabla h|^{2},
$$

where $C_{1}(n, \alpha)$ and $C_{2}(n, \alpha)$ are positive constants.

Proof. We prove this proposition case by case.

(i) The case of $c<0$ and $\alpha>0$. In the proof of Lemma 2.1 we see that by choosing an $\epsilon=\epsilon(n, \alpha) \in(0,1)$ such that $\frac{n+2}{n}(\epsilon-1)+\alpha \geq 0$ we get the

$$
\begin{aligned}
\frac{1}{2} \Delta|\nabla h|^{2} \geq & \left(\frac{(\epsilon-1)^{2}}{n \epsilon^{2}}-\frac{\epsilon-1}{\epsilon}\right) \frac{|\nabla h|^{4}}{h^{2}} \\
& +\frac{\epsilon-1}{\epsilon} \frac{\nabla h}{h} \nabla\left(|\nabla h|^{2}\right)-K|\nabla h|^{2} .
\end{aligned}
$$

Then we see that $C_{1}(n, \alpha)=\frac{(\epsilon-1)^{2}}{n \epsilon^{2}}-\frac{\epsilon-1}{\epsilon}>0$ and $C_{2}(n, \alpha)=\frac{1-\epsilon}{\epsilon}>0$.

(ii) The case of $c>0$ and $\frac{n+2}{2(n-1)}<\alpha<\frac{2 n^{2}+9 n+6}{2 n(n+2)}$ when $n \geq 3$. In this case, (2.5) is equivalent to

$$
\epsilon>1-\frac{n \alpha}{n+2}
$$

We can check

$$
\frac{5 n+6}{2\left(n^{2}+2 n\right)}<\frac{-(n-4)+\sqrt{n^{2}+5 n+3}}{2(n-1)} .
$$

Hence, when $n \geq 3$, for any $\alpha$ satisfies

$$
-\frac{n-4}{2(n-1)}<\alpha-1<\frac{5 n+6}{2\left(n^{2}+2 n\right)}
$$

which is equivalent to

$$
\frac{n+2}{2(n-1)}<\alpha<\frac{2 n^{2}+9 n+6}{2 n(n+2)},
$$

then (2.21) is satisfied by choosing

$$
\epsilon:=\tilde{\epsilon}=\frac{(5 n+6)-2(\alpha-1)\left(n^{2}+2 n\right)}{2\left(n^{2}+5 n+3\right)},
$$

and it is easy to check that $\epsilon \in(0,1)$.

In particular, we let

$$
\delta=\tilde{\delta}:=\frac{1}{2}\left[\frac{n c}{c^{2} \tilde{\epsilon}^{2}}\left(\frac{n+2}{n}(\tilde{\epsilon}-1)+\alpha\right)+\frac{\frac{(\tilde{\epsilon}-1)^{2}}{n \tilde{\epsilon}^{2}}-\frac{\tilde{\epsilon}-1}{\tilde{\epsilon}}}{c\left(\frac{n+2}{n}(\tilde{\epsilon}-1)+\alpha\right)}\right],
$$


then (2.10) and (2.11) are satisfied and (2.7) becomes

$$
\frac{1}{2} \Delta|\nabla h|^{2} \geq \tilde{C}_{1}(n, \alpha) \frac{|\nabla h|^{4}}{h^{2}}-\tilde{C}_{2}(n, \alpha) \frac{\nabla h}{h} \nabla\left(|\nabla h|^{2}\right)-K|\nabla h|^{2},
$$

where positive constants $\tilde{C}_{1}(n, \alpha)$ and $\tilde{C}_{2}(n, \alpha)$ are given by

respectively.

$$
\begin{gathered}
\tilde{C}_{1}(n, \alpha)=\frac{1}{2}\left[\left(\frac{(\tilde{\epsilon}-1)^{2}}{n \tilde{\epsilon}^{2}}-\frac{\tilde{\epsilon}-1}{\tilde{\epsilon}}\right)-\frac{n}{\tilde{\epsilon}^{2}}\left(\frac{n+2}{n}(\tilde{\epsilon}-1)+\alpha\right)^{2}\right], \\
\tilde{C}_{2}(n, \alpha)=\frac{4(\alpha-1) n(n+2)+n(2 n+5)}{(5 n+6)-4(\alpha-1) n(n+2)},
\end{gathered}
$$

We conclude the proof of Proposition 2.3.

Now, we are in a position to prove our Theorem 1.4, Denote by $B_{p}(R)$ the geodesic ball centered at $p$ with radius $R$. Let $\phi$ be a cut-off function (see [9]) satisfying $\operatorname{supp}(\phi) \subset B_{p}(2 R),\left.\phi\right|_{B_{p}(R)}=1$ and

$$
\begin{gathered}
\frac{|\nabla \phi|^{2}}{\phi} \leq \frac{C}{R^{2}} \\
-\Delta \phi \leq \frac{C}{R^{2}}(1+\sqrt{K} R \operatorname{coth}(\sqrt{K} R)),
\end{gathered}
$$

where $C$ is a constant depending only on $n$. We define $G=\phi|\nabla h|^{2}$ and will apply the maximum principle to $G$ on $B_{p}(2 R)$. Moreover, we assume $G$ attains its maximum at the point $x_{0} \in B_{p}(2 R)$ and assume $G\left(x_{0}\right)>0$ (otherwise the proof is trivial). Then at the point $x_{0}$, it holds that

$$
\Delta G \leq 0, \quad \nabla\left(|\nabla h|^{2}\right)=-\frac{|\nabla h|^{2}}{\phi} \nabla \phi
$$

and

$$
\begin{aligned}
0 \geq & \Delta G \\
= & \phi \Delta\left(|\nabla h|^{2}\right)+|\nabla h|^{2} \Delta \phi+2 \nabla \phi \nabla|\nabla h|^{2} \\
= & \phi \Delta\left(|\nabla h|^{2}\right)+\frac{\Delta \phi}{\phi} G-2 \frac{|\nabla \phi|^{2}}{\phi^{2}} G \\
\geq & 2 \phi\left[C_{1}(n, \alpha) \frac{|\nabla h|^{4}}{h^{2}}-C_{2}(n, \alpha) \frac{\nabla h}{h} \nabla\left(|\nabla h|^{2}\right)-K|\nabla h|^{2}\right] \\
& +\frac{\Delta \phi}{\phi} G-2 \frac{|\nabla \phi|^{2}}{\phi^{2}} G \\
= & 2 C_{1}(n, \alpha) \frac{G^{2}}{\phi h^{2}}+2 C_{2}(n, \alpha) \frac{G}{\phi} \nabla \phi \frac{\nabla h}{h}-2 K G+\frac{\Delta \phi}{\phi} G-2 \frac{|\nabla \phi|^{2}}{\phi^{2}} G,
\end{aligned}
$$

where, in the second inequality, the estimate (2.27) is used. Multiplying both sides of (2.30) by $\frac{\phi}{G}$ yields

$$
2 C_{1}(n, \alpha) \frac{G}{h^{2}} \leq-2 C_{2}(n, \alpha) \nabla \phi \frac{\nabla h}{h}+2 \phi K-\Delta \phi+2 \frac{|\nabla \phi|^{2}}{\phi} .
$$


Using the Cauchy inequality

$$
\begin{aligned}
-2 C_{2}(n, \alpha) \nabla \phi \frac{\nabla h}{h} & \leq 2 C_{2}(n, \alpha)|\nabla \phi| \frac{|\nabla h|}{h} \\
& \leq \frac{C_{2}^{2}(n, \alpha)}{C_{1}(n, \alpha)} \frac{|\nabla \phi|^{2}}{\phi}+C_{1}(n, \alpha) \frac{G}{h^{2}},
\end{aligned}
$$

into (2.31) yields

$$
C_{1}(n, \alpha) \frac{G}{h^{2}} \leq 2 \phi K-\Delta \phi+\left(2+\frac{C_{2}^{2}(n, \alpha)}{C_{1}(n, \alpha)}\right) \frac{|\nabla \phi|^{2}}{\phi} .
$$

Hence, for $x \in B_{p}(R)$, we have

$$
\begin{aligned}
C_{1}(n, \alpha) G(x) & \leq C_{1}(n, \alpha) G\left(x_{0}\right) \\
& \leq h^{2}\left(x_{0}\right)\left[2 K+\frac{C(n, p)}{R^{2}}(1+\sqrt{K} R \operatorname{coth}(\sqrt{K} R))\right] .
\end{aligned}
$$

It shows that

$$
|\nabla u|^{2}(x) \leq C(n, \alpha) M^{2}\left[K+\frac{1}{R^{2}}(1+\sqrt{K} R \operatorname{coth}(\sqrt{K} R))\right]
$$

and hence,

$$
|\nabla u(x)| \leq C(n, \alpha) M \sqrt{K+\frac{1}{R^{2}}(1+\sqrt{K} R \operatorname{coth}(\sqrt{K} R))} .
$$

We complete the proof of Theorem 1.4 .

\section{Appendix}

Here we give a sketch of the proof of theorem 1.2. The interested readers can consult Yang's paper [10] for details. Assume that $u(x)$ is a positive solution to (1.3) with $c>0$ and $\alpha \leq 1$. Let $f=\log u$. Then we have

$$
\Delta f=-|\nabla f|^{2}-c u^{\alpha-1} \text {. }
$$

Let $F=|\nabla f|^{2}+c u^{\alpha-1}$. Then we have $\Delta f=-F$ and by the well-known Weitzenbock-Bochner formula

$$
\Delta|\nabla f|^{2}=2 \nabla f \nabla \Delta f+2\left|\nabla^{2} f\right|^{2}+2 \operatorname{Ric}(\nabla f, \nabla f),
$$

where $\nabla^{2} f$ is the Hessian of $f$. Since $c>0$ and $\alpha \leq 1$, we obtain by the above two inequalities

$$
\begin{aligned}
\Delta F & =\Delta|\nabla f|^{2}+c \Delta u^{\alpha-1} \\
& =-2 \nabla f \nabla F+2\left|\nabla^{2} f\right|^{2}+2 \operatorname{Ric}(\nabla f, \nabla f) \\
& +c(1-\alpha) u^{\alpha-1} F+c(1-\alpha)^{2} u^{\alpha-1}|\nabla f|^{2} \\
& \geq-2 \nabla f \nabla F+\frac{2}{n} F^{2}-2 K F
\end{aligned}
$$

on $B_{p}(2 R)$, where we used the fact that $\left|\nabla^{2} f\right|^{2} \geq \frac{1}{n}(\Delta f)^{2}$. Then following Yang's proof line by line we finish the proof of theorem 1.2 . 


\section{REFERENCES}

[1] K. Brighton, A Liouville-type theorem for smooth metric measure spaces, J. Geom. Anal. 23 (2013), 562-570.

[2] B. Gidas, J. Spruck, Global and local behavior of positive solutions of nonlinear elliptic equations, Comm. Pure Appl. Math. 34(1981), no.4, 525-598.

[3] Z. Guo, J. Wei, Hausdoff dimension of ruptures for solutions of a semilinear equation with singular nonlinearity, Manuscripta Math. 120 (2006), 193-209.

[4] G. Y. Huang, B. Q. Ma, Gradient estimates and Liouville type theorems for a nonlinear elliptic equation, Arch. Math. 105 (2015), 491-499.

[5] G. Y. Huang, B. Q. Ma, Hamilton's gradient estimates of porous medium and fast diffusion equations, Geom. Dedicata, 188 (2017), 1-16.

[6] G. Y. Huang, Z. Li, Liouville type theorems of a nonlinear elliptic equation for the $V$-Laplacian, Anal. Math. Phys. DOI 10.1007/s13324-017-0168-6.

[7] J. Y. Li, Gradient estimate for the heat kernel of a complete Riemannian manifold and its applications, J. Funct. Anal. 97 (1991) 293-310.

[8] B. Qian, Yau's gradient estimates for a nonlinear elliptic equation, Arch. Math. 108 (2017), 427-435.

[9] R. Schoen, S.-T. Yau, Lectures on Differential Geometry, International Press, 1994.

[10] Y. Y. Yang, Gradient estimates for the equation $\Delta u+c u^{-\alpha}=0$ on Riemannian manifolds, Acta Math. Sin. (Engl Ser), 26 (2010), 1177-1182.

[11] S. T. Yau, Harmonic functions on complete Riemannian manifolds, Comm. Pure Appl. Math. 28 (1975), 201-228.

Department of Mathematics, Henan Normal University, XinXiang 453007, P.R. CHINA,

E-mail address: bqma@henannu.edu.cn

Department of Mathematics, Henan Normal University, Xinxiang 453007, P.R. CHINA,

E-mail address: hgy@henannu.edu.cn

SCHOOL OF MATHEMATiCs AND Statistics, WuHAN UNiversity, WuHAN 430072, P.R. China,

E-mail address: yongluo@whu.edu.cn 